\section{Assessment of a new hydrophilic acrylic supplementary IOL for sulcus fixation in pseudophakic cadaver eyes}

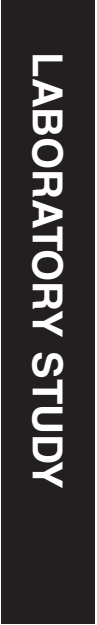

'Department of Ophthalmology and Visual Sciences, John A. Moran Eye Center, University of Utah, Salt Lake City, UT, USA

${ }^{2}$ Department of Ophthalmology, University Hospital Ayr, Ayr, UK

Correspondence: L Werner, Department of Ophthalmology and Visual Sciences, John A. Moran Eye Center, University of Utah, 65 Mario Capecchi Drive, Salt Lake City, UT 84132, USA

Tel: +1 8015816586 ;

Fax: +1 8015813357

E-mail: liliana.werner@

hsc.utah.edu

Received: 27 April 2016 Accepted: 30 November 2016;

Published online:

20 January 2017

\author{
Abstract \\ Purpose Management of refractive errors \\ after cataract surgery includes spectacles or \\ contact lens, secondary laser vision correction, \\ intraocular lens (IOL) exchange, or piggyback \\ lens implantation. We evaluated for the first \\ time a single-piece hydrophilic acrylic IOL \\ designed for supplementary sulcus fixation in \\ postmortem pseudophakic human eyes. \\ Methods Pseudophakic human cadaver eyes \\ were imaged by anterior segment optical \\ coherence tomography (AS-OCT) to assess \\ position of the primary IOL. Eyes were \\ prepared as per the Miyake-Apple technique. \\ The supplementary IOL (Medicontur A4 \\ Addon IOL family) was then inserted into the \\ ciliary sulcus. AS-OCT and photographs from \\ anterior and posterior views were used to \\ assess IOL centration, tilt, and interlenticular \\ distance from the primary IOL. \\ Results Data were obtained from 12 eyes \\ having primary IOLs of varying materials and \\ designs in the bag and representing different \\ sizes of eyes and severity of Soemmering's \\ ring formation. The A4 Addon IOL was \\ successfully inserted into the ciliary sulcus \\ and was well centered in all cases. Four cases \\ of tilt were observed on AS-OCT: three with \\ mild tilt due to pre-existing zonular \\ dehiscence, and one due to a localized area of \\ Soemmering's ring formation. Interlenticular \\ distance ranged from 0.34 to $1.24 \mathrm{~mm}$ and was \\ not dependent on severity of Soemmering's \\ ring or type of primary IOL. \\ Conclusions The A4 Addon IOL was \\ designed for sulcus fixation as a \\ supplementary lens, with a large diameter, \\ a square-shaped optic, four smooth loop \\ haptics, and a convex-concave optical surface. \\ It exhibited appropriate centration and
}

N Reiter ${ }^{1}$, L Werner ${ }^{1}$, J Guan ${ }^{1}$, J Li ${ }^{1}$, KT Tsaousis ${ }^{1}$, $N$ Mamalis ${ }^{1}$ and S Srinivasan ${ }^{2}$ interlenticular distance with different primary in-the-bag IOLs.

Eye (2017) 31, 802-809; doi:10.1038/eye.2016.310; published online 20 January 2017

\section{Introduction}

Residual refractive error remains a major cause of patients' dissatisfaction after cataract surgery and still represents a significant cause for intraocular lens (IOL) explantation or secondary intervention. ${ }^{1}$ Even with modern biometry methods, refractive surprises due to incorrect IOL power calculations still occur, on the order of $45 \%$ of cases in one large series. ${ }^{2}$ Management of refractive errors after cataract operations includes spectacles or contact lens, secondary laser vision correction, IOL exchange, or piggyback lens implantation. As patients have high expectations preferring spectacle independence and given that the cost and risks of a laser operation or an IOL explantation are considerable factors, piggyback implantation offers a viable alternative especially in cases of extreme ametropia and corneal abnormalities. ${ }^{3}$

Piggyback implantation was first reported 1993 with the 'two-in-the-bag' technique. ${ }^{4}$ This technique was used throughout the 1990s until it was discovered that placing two lenses in the bag allowed an avenue for lens epithelial cells to proliferate, resulting in interlenticular opacification (ILO). ${ }^{5-7}$ Cataract surgeons then began to place IOLs in a piggyback configuration in the sulcus. However, as these lenses were designed to be placed in the capsular bag, their sharp edges and rough sidewalls (designed to prevent posterior capsule opacification and positive dysphotopsia) excoriated the posterior iris, resulting in pigment 
dispersion syndrome (PDS) and thus pigmentary glaucoma in some cases. ${ }^{8}$ Since 2007 , several piggyback IOLs with optimized designs for sulcus implantation have been introduced: the Sulcoflex (Rayner Intraocular Lenses Ltd, Hove, United Kingdom) ${ }^{9}$ the Aspira (HumanOptics AG, Erlangen, Germany), ${ }^{10}$ and the recently available A4 AddOn IOL (Medicontur Medical Engineering Ltd, Zsámbék, Hungary/1stQ GmbH, Mannheim, Germany). These models share most of the following design features that are considered to be optimal for sulcus implantation: round optic edges; shallow anterior curvature; posterior haptic angulation (except the A4 Addon IOL presenting zero angulation); and thin haptics to minimize iris chafing, concave posterior surface to avoid contact with the primary IOL, and a large optic diameter to prevent pupillary capture. The A4 Addon further presents a square-shaped optic that, according to the manufacturer, was designed to avoid pupillary capture.

The aim of this study was to assess the new hydrophilic A4 AddOn IOL family (models A45D and A45SML, Medicontur Medical Engineering Ltd, Zsámbék, Hungary/1stQ GmbH, Mannheim, Germany) that incorporates the above-mentioned characteristics for sulcus fixation. Our primary focus was the use of a cadaver eye model to evaluate parameters significant for its clinical behavior: IOL fixation; centration; tilt; and clearance relative to the primary IOL. The study model is associated with limitations that does not allow for accurate assessment of the direct interaction between the study lens and the dynamic physiological iris in human eyes.

\section{Materials and methods}

The new add-on IOL is a single-piece hydrophilic acrylic lens manufactured from a material with $25 \%$ water content, containing an ultraviolet light absorber. The aspheric optic of the lens has an overall square shape, with an effective diameter of $6.0 \mathrm{~mm}$, and a convexconcave configuration. Attached to the optic are four flexible closed loops with a thickness of $0.3 \mathrm{~mm}$. The overall diameter of the lens is $13.0 \mathrm{~mm}$. Standard powers range from -10.0 to $+10.0 \mathrm{D}$ in $0.25 \mathrm{D}$ increments; other powers are available upon request (Figure 1).

This study was performed in collaboration with eye banks nationwide within the United States. Pseudophakic human eyes obtained postmortem were immersed in 10\% neutral buffered formalin upon enucleation. Once available in our laboratory, gross measurements of each eye were obtained by using a digital metric ruler (model Absolute Digimatic, Mitutoyo Corp., Kawasaki, Japan), including anterior-posterior length, equatorial diameter, and corneal diameter. The eyes were then bisected coronally just anterior to the equator. Gross examination and photographs of the anterior segment were done from the posterior aspect (Miyake-Apple view) to assess the type and fixation of the IOL implanted, as well as the degree of Soemmering's ring formation. Soemmering's ring (related to proliferative/regenerative material within the equatorial region of the capsular bag, outside of the optic), had a score of intensity (SRI) from 0 to 4 , and a score of area (SRA), related to the number of quadrants involving the highest intensity. Only eyes with in-the-bag fixated IOLs were selected for inclusion in the study. The anterior segment of each selected eye was then glued to a
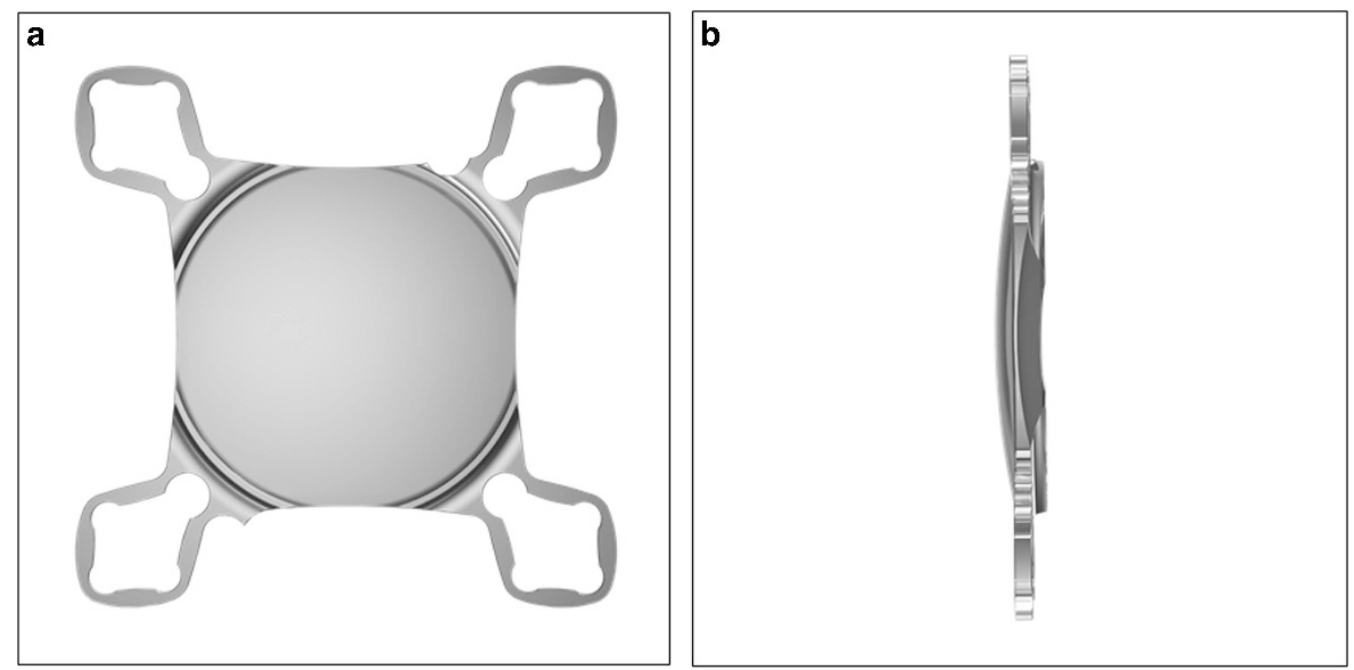

Figure 1 Computer-generated images showing the overall design of the hydrophilic acrylic supplementary IOL evaluated in this study. (a) Anterior view. (b) Side view. 
மூ 舟茴

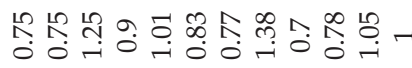
(1)

honmththathenth $m 0 \operatorname{man} m \operatorname{mathm}$

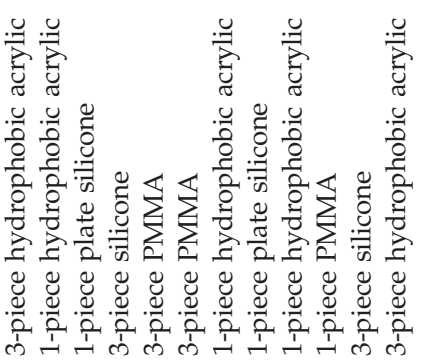

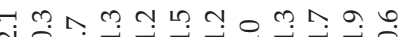

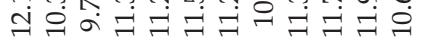

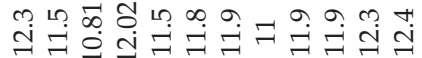

m म

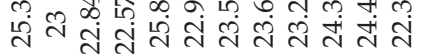
|

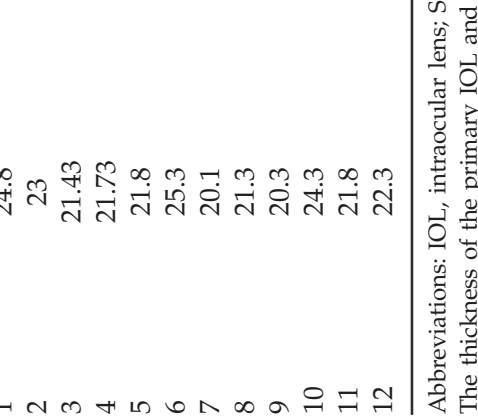

glass slide according to the Miyake-Apple technique for experimental surgery, ${ }^{11}$ and underwent pre-operative examination under the Visante anterior segment optical coherence tomography (Visante, AS-OCT; Carl Zeiss Meditec, Jena, Germany) to assess centration, tilt, and thickness of the primary IOL.

The cornea was then removed with scissors. The iris was also partially removed for $360^{\circ}$ with scissors to improve visualization in these postmortem eyes in which pupillary dilation is not possible; a peripheral rim of iris was kept to simulate a pupil dilation of $\sim 8.0 \mathrm{~mm}$. Ophthalmic viscosurgical device (OVD) was injected behind the iris. The add-on lens was then inserted behind the iris with forceps, and positioned in the sulcus with a hook. Residual OVD was then removed by irrigation and aspiration (Infinity, Alcon, Fort Worth, TX, USA). The eyes underwent post-implantation evaluation with the Visante AS-OCT. The remaining peripheral iris was then completely removed and the position of each closed loop haptic element was analyzed under the operating microscope. Photographs were obtained for documentation. The analyses focused on the fixation, centration, and tilt of the add-on lens, as well as on the interlenticular distance (ILD) between the primary IOL and the add-on lens.

\section{Results}

Twelve eyes were selected for inclusion in this study based on gross measurements, the type of primary in-thebag implanted IOL, as well as the degree of Soemmering's ring formation. Eyes were chosen so as to have a wide variety of sizes, primary IOL materials and designs, and degrees of Soemmering's ring formation in order to replicate the many possible clinical scenarios that the study add-on lens might encounter. Table 1 shows the overall characteristics of the eyes included. The mean age of the donors was 88.6 years. The mean axial length, horizontal equatorial diameter, and horizontal corneal diameter of the eyes were $22.35 \pm 1.68,23.64 \pm 1.09$, and $11.77 \pm 0.49 \mathrm{~mm}$, respectively. The following primary IOLs were represented: 3-piece hydrophobic acrylic $(N=2), 1$-piece hydrophobic acrylic $(N=3), 1$-piece plate silicone $(N=2)$, 3-piece silicone $(N=2)$, 3-piece PMMA $(N=2)$, and 1-piece PMMA $(N=1)$. Soemmering's ring formation ranged from 0 (zero) to intensity 4 in 4 quadrants (SRI $\times$ SRA ranged from 0 to 16 ). It is noteworthy that 1 eye implanted with a 1-piece plate silicone IOL (eye No. 3) had Soemmering's ring formation localized to 1 quadrant. Three other eyes (eyes No. 4, 6, 8) exhibited localized areas of zonular dehiscence under gross evaluation. Figure 2 shows Miyake-Apple views of some of the eyes included in this study. 

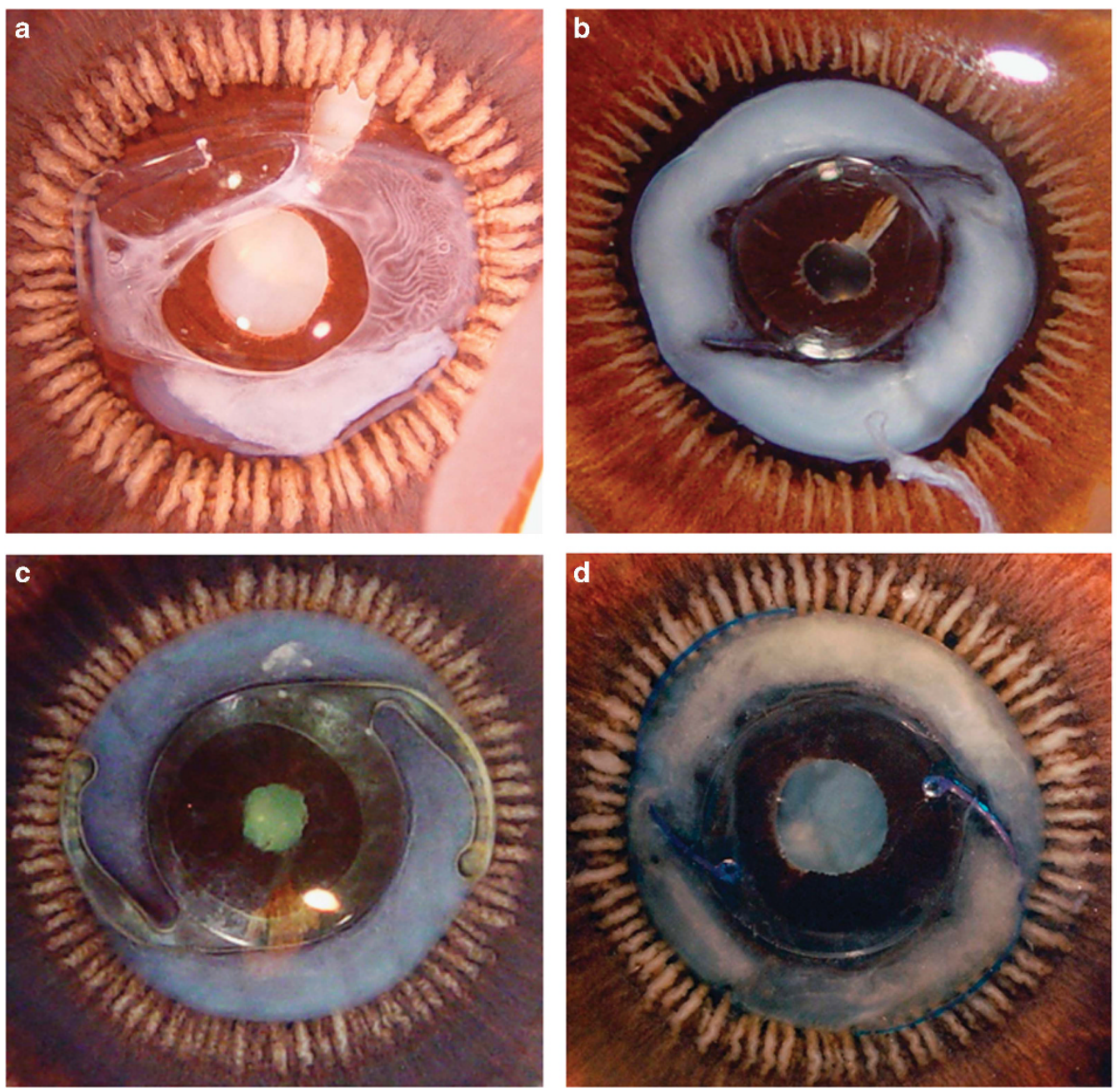

Figure 2 Posterior Miyake-Apple views of four eyes included in this study, displaying the variety of IOL type and Soemmering's ring severity. (a) 1-piece plate silicone with moderate Soemmering's ring (eye No. 3). (b) 3-piece PMMA with severe Soemmering's ring (eye No. 6). (c) 1-piece hydrophobic acrylic with moderate Soemmering's ring (eye No. 7). (d) 3-piece silicone with severe Soemmering's ring (eye No. 11).

The study add-on lenses were found to be well centered in all eyes, and no tilt was observed in the majority of them (Figure 3). Some tilt of the add-on lens in relation to the primary in-the-bag IOL was observed under AS-OCT examination in some cases. Three of them were in the eyes with localized areas of zonular dehiscence (eyes No. 4, $6,8)$. The tilt observed in these eyes was mild. Gross evaluation revealed that at least one of the loop haptics was located posterior to the ciliary processes through a localized area of zonular dehiscence, while the other loops were fixated in the sulcus (Figure 4).

A fourth case of tilt was observed in the eye with localized Soemmering's ring formation (eye No. 3). Tilt was observed when the add-on IOL was initially positioned such that one of the loops was directly overlying the Soemmering's ring anteriorly while the remaining three loops were positioned free of any
Soemmering's ring. The tilt resolved upon rotation of the add-on lens so that no loop was overlying the localized Soemmering's ring.

In all cases, an interlenticular space was observed between the primary-in-the-bag IOL and the add-on lens. The ILD between the study lens and the primary IOL (measured in the center) was always greater than $0.34 \mathrm{~mm}$ (average: $0.68 \mathrm{~mm}$; range: $0.34-1.24 \mathrm{~mm}$; Table 1). We could not demonstrate a correlation between the ILD and Soemmering's ring formation (SRI $\times$ SRA), or with the thickness of the primary IOL (correlation coefficient $(r)$ : -0.02 and 0.06$)$.

\section{Discussion}

The past decade has seen significant improvements in the design characteristics of supplementary IOLs. Given that 
patients' expectation are quite high for spectacle free vision, this type of secondary intervention offers a valuable alternative for optical enhancement after cataract
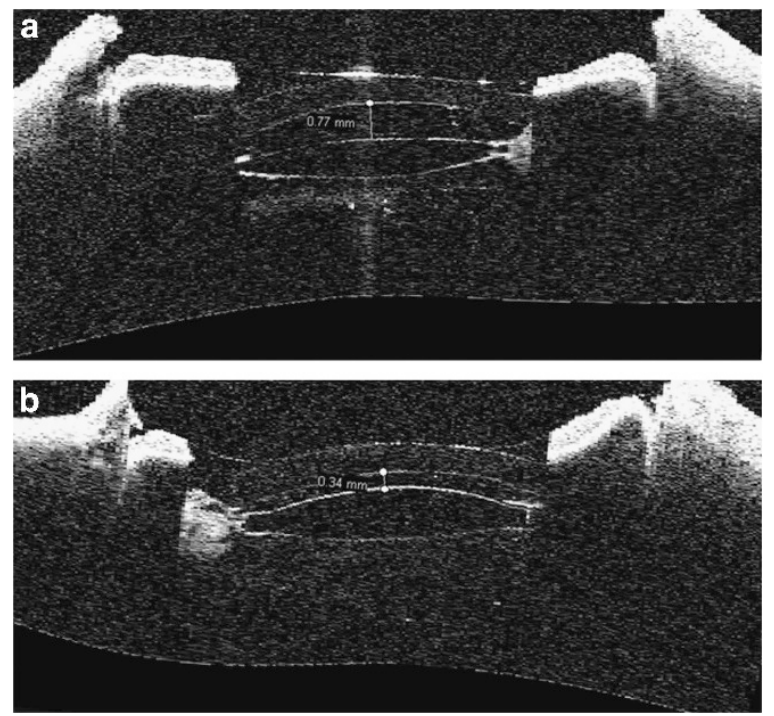

Figure 3 Anterior OCT images of two eyes showing no tilt of the add-on lens relative to the primary IOL. (a) 1-piece PMMA IOL in the bag with severe Soemmering's ring; $0.77 \mathrm{~mm}$ interlenticular distance (eye No. 10). (b) 3-piece silicone IOL in the bag with moderate/severe Soemmering's ring; $0.34 \mathrm{~mm}$ interlenticular distance (eye No. 11). surgery; whether it be to correct a refractive surprise or to add a premium function such as multifocality or toricity to the primary IOL. ${ }^{12-16}$ The study lenses included premium characteristics like diffractive optic (A45D) or extra addition for macular diseases (A45SML), whereas toric features are also commercially available on the same IOL platform. However, the optical properties were not relevant in our study.

Although PRK, LASIK, and IOL exchange techniques offer better refractive 'fine tuning' to within $0.1 \mathrm{D}$ while realistic expectations for piggyback implantation are to within $0.25 \mathrm{D}$, laser refractive surgeries are not traditionally covered by health care providers and laser vision correction in this group of patients may lead to chronic dry eye. ${ }^{17,18}$ In addition, patients with thin corneas that cannot undergo refractive laser surgery or patients in areas where this service is unavailable may be good candidates for piggyback implantation. Lens exchange can be a risky procedure in the case of a fibrotic capsule, with the IOL firmly attached to a phimotic capsular bag. IOL exchange can be considered if the IOL is malpositioned, scratched, or otherwise compromised. However, if the issue is due to incorrect IOL power selection, one must know the cause of refractive surprise if lens exchange will be performed (eg, incorrect IOL used, biometry issues). With a piggyback lens, the cause
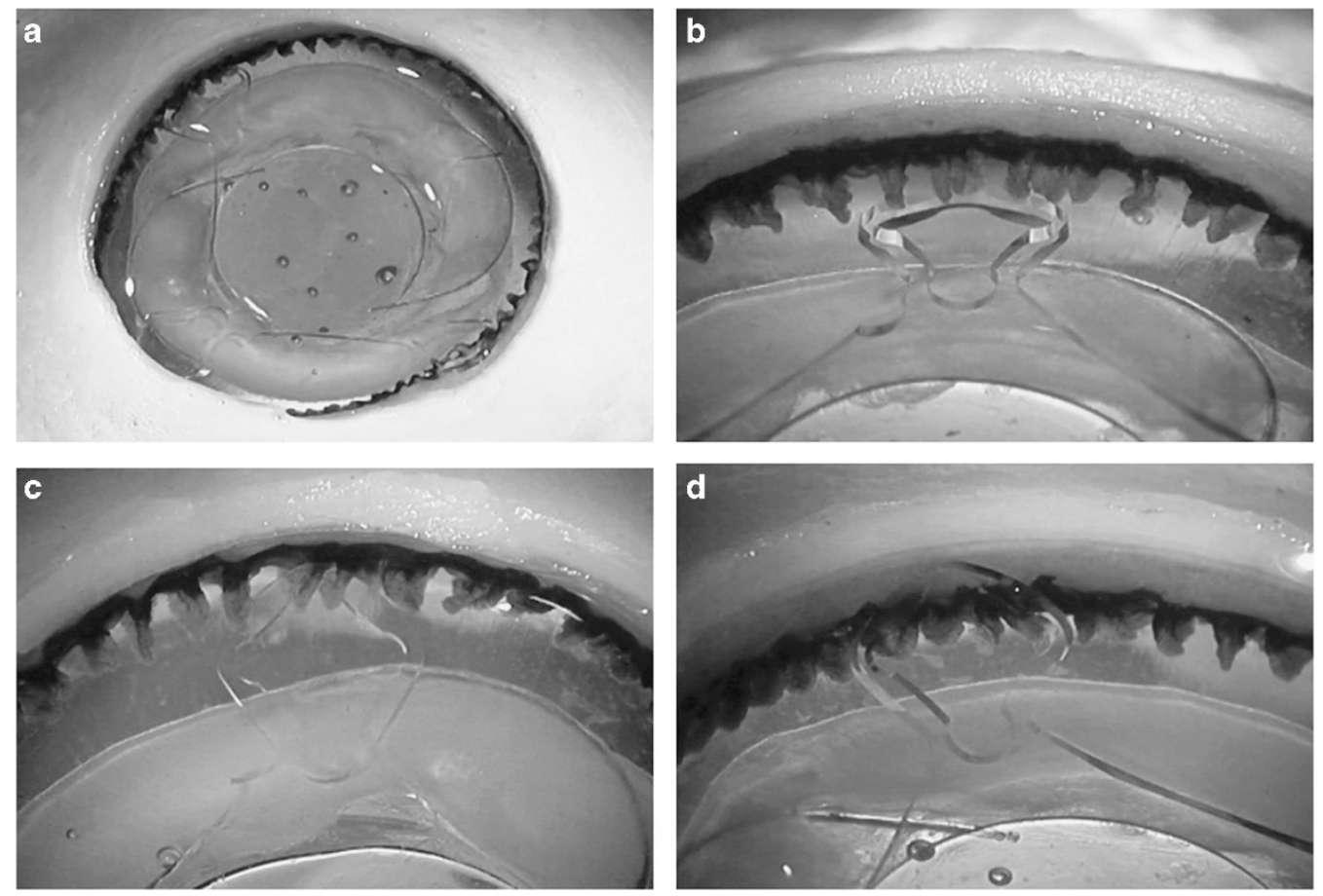

Figure 4 Anterior photographs of eye No. 6, in which mild tilt of the add-on lens was observed on OCT due to an area of zonular dehiscence. (a) The loop haptic at 11 o'clock can be seen posterior to the ciliary processes through a localized area of zonular dehiscence. (b) A closer view of the same loop haptic, located posterior to the ciliary processes due to compromised zonules. (c and d): the other haptics were correctly positioned in the ciliary sulcus. 
of the refractive surprise does not have to be known; rather, only the corneal thickness and current refraction are needed, and an add-on lens can be reliably placed to correct the patient's refraction. Piggyback implantation is a far easier procedure than IOL exchange, and is easily reversed. IOL exchange can be very technical, and carries the risk of capsular rupture (about $8 \%$ of cases) and zonular dehiscence (about $50 \%$ of cases). ${ }^{19-21}$ Apart from the risk, a prospective case series of 23 patients reported $92 \%$ of eyes that underwent piggyback lens implantation resulted in refraction to within $0.5 \mathrm{D}$ of the intended correction, whereas only $82 \%$ of eyes that underwent IOL exchange achieved this goal. ${ }^{22}$ This discrepancy was perhaps due to the exchanged IOL being placed in a different plane than the original IOL. Another advantage of piggyback implantation is its reversibility, which can be useful in cases of post-op astigmatism after keratoplasty, in which a toric IOL can be temporarily placed in the sulcus, or in patients with prolonged silicone oil tamponade.

The scope of our study was to demonstrate the fit of this new supplementary IOL in cadaver eyes, as appropriate alignment is important for not inducing optical aberrations, which will compromise the patient's visual acuity. One significant parameter is the interlenticular distance-ILD: the distance between the supplementary and the primary IOL. Contact between IOLs can potentially result in opacification of the interface. In our study, in all cases a satisfactory ILD was found (range: $0.34-1.24 \mathrm{~mm}$ ). This distance is likely adequate to prevent opacification as our laboratory previously reported the Rayner Sulcoflex to have a smaller ILD of $0.23-0.78 \mathrm{~mm}$ also in cadaver eyes, ${ }^{23}$ and to our knowledge there do not exist any reports in the literature of ILO with the Sulcoflex lens. Like the add-on lenses currently on the market, the study lens has a convex-concave configuration in order to maximize the ILD. It is noteworthy that the ILD values reported in this study and in the study on the Sulcoflex lens were obtained in cadaver eyes, and likely do not represent the actual ILD values that would be observed clinically, considering the limitations of the study model. However, the results showed the presence of clearance between inthe-bag and add-on IOLs in all cases.

The lens further presents a rim on the posterior optic edge, designed to keep the distance to the primary bag lens with four discontinuations to ensure the uninterrupted flow of aqueous humor, according to the manufacturer. The IOL's rounded edges have the potential to minimize iris chafing in order to prevent iris pigment dispersion; subsequently, intraocular pressure will likely be stable. This is, however, a parameter that could not be assessed in our study. Also, the interaction between the study lens and the posterior surface of the iris could not be accurately assessed in this study due to postmortem iris collapse. The study lens has zero degrees haptic angulation while the Sulcoflex has $10^{\circ}$. This explains why, as previously mentioned, the study lens exhibited a greater ILD than the Sulcoflex relative to the primary IOL in cadaver eyes. ${ }^{23}$ Our current results did not demonstrate a correlation between ILD and severity of Soemmering's ring, and also no correlation of ILD with primary IOL thickness, which may be due to the relatively small sample size. In a similar cadaver eye study evaluating the Sulcoflex, the ILD appeared mostly dependent on the primary IOL, but it is not known if the correlation was strong as the thickness of the primary IOL was not measured in that study. ${ }^{23}$ By using the Soemmering's ring data and ILD data from the same above-mentioned Sulcoflex study, the correlation coefficient $(r)$ was calculated as -0.20 .

An important issue with the study lens is the possible tilting relative to the primary IOL that may occur after implantation. Our results highlighted two main mechanisms that can lead to lens tilt: (1) localized Soemmering's ring formation and (2) pre-existing zonular dehiscence. In the former, severe and markedly asymmetric cortical proliferation in the capsular bag pushes one haptic of the supplementary IOL anteriorly, creating an angle between the $X Y$ plane and the lens. This finding was evident in one case of our study, but the tilt was completely resolved after a $45^{\circ}$ rotation of the IOL in the $X Y$ plane such that the localized Soemmering's ring and haptics no longer overlapped. The second potential factor that can lead to lens tilt is zonular weakness or dehiscence, which allows an avenue for one of the loop haptics to dislocate posterior to the ciliary processes and causing additional stress to the zonules. Given these two observed causes of add-on lens tilt, we advise performing a meticulous preoperative examination through AS-OCT and/or ultrasound biomicroscopy in order to reveal possible asymmetric Soemmering's ring. Zonular dehiscence is more difficult to determine pre-operatively, but a shallow anterior chamber in an otherwise normal axial length eye can be a clue.

To conclude, in this study with pseudophakic cadaver eyes of varying axial lengths, severity levels of cortical proliferation in the capsular bag, and types of primary IOLs, the study lens A45D/A45SML demonstrated proper fixation and centration in the ciliary sulcus. Adequate distance from the primary IOL was found in all cases and no tilt was observed save for three cases of preexisting zonular dehiscence and one case of localized Soemmering's ring. Although the cadaver eye model used has limitations related to postmortem changes and tissue fixation, it shows the potential of the study lens to exhibit appropriate fit into different pseudophakic eyes. Consequently, we believe the A4 Addon IOL (models A45D/A45SML) appears to be a viable option for clinical 
use, and this lens deserves further investigation of its biocompatibility and optical performance.

\section{Summary}

What was known before

- Biocompatibility of hydrophilic acrylic IOL materials has been thoroughly confirmed in the past.

- Piggyback lens implantation offers several advantages compared with other alternatives for post-cataract surgery refractive surprises.

- Design features specifically for sulcus implantation reduce the incidence of interlenticular opacification, pigmentary dispersion, and pupillary capture.

- Suitable fixation of supplementary IOLs is essential to optimize their clinical performance.

\section{What this study adds}

- This new one-piece hydrophilic acrylic supplementary IOL demonstrated appropriate fixation when implanted in cadaver eyes.

- Two main factors that may cause piggyback IOL tilting are pre-existing asymmetrical cortical proliferation (localized Soemmering's ring) and preexisting zonular dehiscence.

\section{Conflict of interest}

LW reports research grants from Medicontur, during the conduct of the study; grants from Abbott Medical Optics, grants from AcuFocus, grants from Alcon, grants from Anew Optics, grants from Bausch and Lomb, grants from ClarVista, grants from Hoya, grants and personal fees from PowerVision, grants from Genisphere, grants from LensGen, grants from Mynosys, grants from Omega, grants from Sharklet, outside the submitted work. NM reports research grants from Medicontur, during the conduct of the study; grants from Abbott Medical Optics, grants from Alcon Laboratories, grants from Allergan, grants and other from Anew Optics, grants from Bausch and Lomb, grants from Calhoun Vision Inc, grants from Hoya, grants from Genisphere, grants from Sharklet, grants from ClarVista, grants from LensGen, grants from Omega, grants from Mynosys, grants from PowerVision, outside the submitted work. The remaining authors declare no conflict of interest.

\section{Acknowledgements}

Michael Anderson, COA, Moran Eye Center assisted with the AS-OCT examination. Supported in part by an unrestricted grant from Research to Prevent Blindness, Inc, New York, NY, USA to the Department of Ophthalmology and Visual Sciences, University of Utah, and by a research grant from Medicontur Medical Engineering Ltd., Hungary.

\section{References}

1 Mamalis N, Brubaker J, Davis D, Espandar L, Werner L. Complications of foldable intraocular lenses requiring explantation or secondary intervention-2007 survey update. J Cataract Refract Surg 2008; 34(9): 1584-1591.

2 Behndig A, Montan P, Stenevi U, Kugelberg M, Zetterström C, Lundström M. Aiming for emmetropia after cataract surgery: Swedish National Cataract Register study. J Cataract Refract Surg 2012; 38(7): 1181-1186.

3 Alio JL, Abdelghany AA, Fernández-Buenaga R. Management of residual refractive error after cataract surgery. Curr Opin Ophthalmol 2014; 25(4): 291-297.

4 Gayton JL, Apple DJ, Peng Q, Visessook N, Sanders V, Werner $\mathrm{L}$ et al. Interlenticular opacification: clinicopathological correlation of a complication of posterior chamber piggyback intraocular lenses. J Cataract Refract Surg 2000; 26(3): 330-336.

5 Werner L, Apple DJ, Pandey SK, Solomon KD, Snyder ME, Brint SF et al. Analysis of elements of interlenticular opacification. Am J Ophthalmol 2002; 133(3): 320-326.

6 Micheli T, Cheung LM, Sharma S, Assaad NN, Guzowski M, Francis IC et al. Acute haptic-induced pigmentary glaucoma with an AcrySof intraocular lens. J Cataract Refract Surg 2002; 28(10): 1869-1872.

7 Chang WH, Werner L, Fry LL, Johnson JT, Kamae K, Mamalis N. Pigmentary dispersion syndrome with a secondary piggyback 3-piece hydrophobic acrylic lens. Case report with clinicopathological correlation. J Cataract Refract Surg 2007; 33(6): 1106-1109.

8 Iwase T, Tanaka N. Elevated intraocular pressure in secondary piggyback intraocular lens implantation. J Cataract Refract Surg 2005; 31(9): 1821-1823.

9 Falzon K, Stewart OG. Correction of undesirable pseudophakic refractive error with the Sulcoflex intraocular lens. J Refract Surg 2012; 28(9): 614-619.

10 Basarir B, Kaya V, Altan C, Karakus S, Pinarci EY, Demirok A. The use of a supplemental sulcus fixated IOL (HumanOptics Add-On IOL) to correct pseudophakic refractive errors. Eur J Ophthalmol 2012; 22(6): 898-903.

11 Pereira FaS, Werner L, Milverton EJ, Coroneo MT. MiyakeApple posterior video analysis/photographic technique. J Cataract Refract Surg 2009; 35(3): 577-587.

12 Clare G, Bloom P. Bilateral ciliary sulcus implantation of secondary piggyback multifocal intraocular lenses. J Cataract Refract Surg 2007; 33(2): 320-322.

13 Alfonso JF, Fernández-Vega L, Baamonde MB. Secondary diffractive bifocal piggyback intraocular lens implantation. J Cataract Refract Surg 2006; 32(11): 1938-1943.

14 Meyer JJ, McGhee CN. Supplementary, sulcus-fixated intraocular lens in the treatment of spherical and astigmatic refractive errors in pseudophakic eyes after keratoplasty. Cornea 2015; 34(9): 1052-1056.

15 Žiak P, Šesták M, Mojžiš P, Piñero DP. Piggyback with toric intraocular lens after corneal melting in autoimmune necrotizing vasculitis. Can J Ophthalmol 2013; 48(3): e48-e50.

16 Huerva V. Piggyback multifocal IOLs for a hyperopicpresbyopic surprise after cataract surgery in high myopic patients. Cont Lens Anterior Eye 2014; 37(1): 57-59.

$17 \mathrm{Xu} \mathrm{Y,} \mathrm{Yang} \mathrm{Y.} \mathrm{Dry} \mathrm{eye} \mathrm{after} \mathrm{small} \mathrm{incision} \mathrm{lenticule}$ extraction and LASIK for myopia. J Refract Surg 2014; 30(3): 186-190. 
18 Vestergaard AH, Grauslund J, Ivarsen AR, Hjortdal JO. Efficacy, safety, predictability, contrast sensitivity, and aberrations after femtosecond laser lenticule extraction. J Cataract Refract Surg 2014; 40(3): 403-411.

19 Dagres E, Khan Ma, Kyle GM, Clark D. Perioperative complications of intraocular lens exchange in patients with opacified Aqua-Sense lenses. J Cataract Refract Surg 2004; 30(12): 2569-2573.

20 Lee SJ, Sun HJ, Choi KS, Park SH. Intraocular lens exchange with removal of the optic only. J Cataract Refract Surg 2009; 35(3): 514-518.
21 Altaie R, Loane E, O'Sullivan K, Beatty S. Surgical and visual outcomes following exchange of opacified Hydroview intraocular lenses. Br J Ophthalmol 2007; 91(3): 299-302.

22 El Awady HE, Ghanem AA. Secondary piggyback implantation versus IOL exchange for symptomatic pseudophakic residual ametropia. Graefes Arch Clin Exp Ophthalmol 2013; 251(7): 1861-1866.

23 McIntyre JS, Werner L, Fuller SR, Kavoussi SC, Hill M, Mamalis N. Assessment of a single-piece hydrophilic acrylic IOL for piggyback sulcus fixation in pseudophakic cadaver eyes. J Cataract Refract Surg 2012; 38(1): 155-162. 